\title{
THE GENETICAL ARCHITECTURE OF STERNOPLEURAL CHAETA NUMBER IN DROSOPHILA MELANOGASTER
}

\author{
R. J. KILLICK* \\ Department of Genetics, University of Birmingham, Birmingham B15 2TT
}

Received 4.iii.72

\section{InTRODUCTION}

THE existence of marked inversion stocks of Drosophila melanogaster makes possible the construction of chromosome substitution lines, whose exact chromosomal composition is known. Such lines are especially useful for building up an overall picture of the genetical architecture of a quantitative character or for assessing particular chromosomes in a variety of genotypic backgrounds (Cooke and Mather, 1962; Keller and Mitchell, 1962, 1964; Hill, 1964; Kearsey and Kojima, 1967).

This paper briefly describes an investigation into the genetical architecture of sternopleural chaeta number which confirms previous work and also provides information on genotype-environmental interaction at the chromosomal level.

\section{Materials, methods AND nOtation}

Two sets of chromosome substitution lines were made up; one between the Birmingham strains of Oregon and $6 \mathrm{Cl}$ and the other between the strains of Samarkand and $6 \mathrm{Cl}$. The inversion stocks used in the crossing procedure were $\mathrm{C} 23(\mathrm{a})$ and $\mathrm{Cl}(\mathrm{b})$ which have the constitutions:

$$
\begin{aligned}
& \text { C23(a) Ins (2L + 2R) } \mathrm{Cy} \text {; Cy cn }{ }^{2} \quad \text { In (3LR) } \mathrm{Dc} \times \mathrm{F} \text {; D } \\
& \text { In (2LR) } \mathrm{Pm} \mathrm{al}^{4} \mathrm{ds}^{33 \mathrm{~K}} \mathrm{ltbw}^{4} \quad \mathrm{Sb} \\
& \text { Cl(b) } \quad \mathrm{sg}^{3} / \mathrm{y}^{31 \mathrm{~d}} \mathrm{sc}^{8} \mathrm{dm} \mathrm{B}
\end{aligned}
$$

This ensured that the first three chromosomes, but not the fourth, were controlled and it was assumed that the $\mathrm{Y}$ chromosome was genetically inactive. The efficiency of the chromosome extraction procedure was tested by crossing complementary pairs of substitution lines $(e . g$. OOC $\times \mathrm{CCO}$; where each letter symbolises the origin of the first, second and third chromosome respectively) to give a set of fully heterozygous $F_{1}$ 's all of which should have the same mean. The substitution lines OOO, CCC and SSS were also compared with the original inbred lines. These tests suggested that the substitution lines were fully satisfactory.

In making up all possible genotypes (27 for females; 18 for males) the procedure used was similar to that of Kearsey and Kojima (1967), so that as far as possible each line was used with equal frequency as both male and female parent. Duplicate cultures of all genotypes were made up and raised at $18^{\circ} \mathrm{C}$ and $25^{\circ} \mathrm{C}$. Ten female and ten male progeny were scored from each culture.

* Present address: Scottish Plant Breeding Station, Pentlandfield, Roslin, Midlothian. 
The parameters studied were based on the F $\infty$ metric of van der Veen (1959).

\section{Results}

There were highly significant differences between the genotypes in each of the eight sets ( 2 sexes $\times 2$ temperatures $\times 2$ paris of lines). The parameters describing the effects of each chromosome and all their interactions were estimated by least squares (this is referred to as the full model). All nonsignificant parameters were eliminated and those remaining were reestimated. Further adjustments to the models were made until in the final models the parameters were significant and adequately described the data.

Table 1 shows the summed effects of additive and non-additive parameters from the full and final models of each set. This suggests that: $(a)$

TABLE 1

Summation of additive and non-additive parameters in full and final models

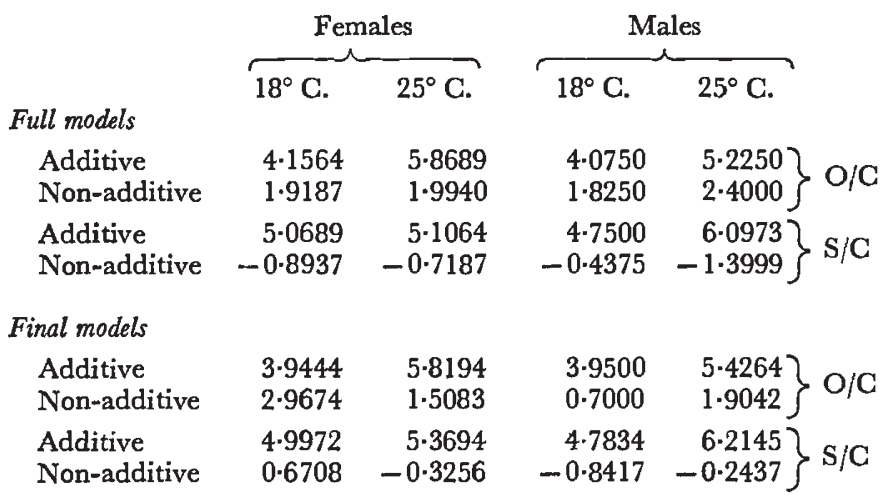

The total genetic effect of the additive parameters was much greater than that of the non-additive parameters. (b) The total effect of the additive parameters was always greater at $25^{\circ} \mathrm{C}$. than at $18^{\circ} \mathrm{C}$. It will be seen later, however, that this effect was not uniform over all chromosomes. (c) The sum of all the non-additive parameters was positive for Oregon/6 $\mathrm{Cl}$ but, with one exception, negative for Samarkand/6 Cl.

In the full models the numbers of negative and positive non-additive parameters were about equal whereas the additive parameters were by definition predominantly positive. These are summarised in table 2 together with a $\chi^{2}$ testing the equality of negative and positive non-additive parameters. In no case did the numbers differ significantly, suggesting that the dominance and epistasis were ambidirectional. The last two columns demonstrate this effect; the penultimate column gives the absolute total effect of the non-additive parameters regardless of sign and the last column gives the net effect when sign is taken into account. Quite clearly the net effect was a fraction of the potential if the non-additivity was directional. In the lower part of table 2 the parameters are treated separately. Of the four non-additive parameters, $h, j$ and $l$ were all approximately equally positive and negative and the effect of this ambidirectional nature was to reduce the total possible contribution to less than a tenth. The $i$ parameter, 
however, was more frequently positive than negative and although the potential effect was reduced it was not nearly as marked as with the other non-additive parameters. The last row of table 2 shows the additive parameters considered alone. In only one instance was there a negative effect $\left(d_{2}\right.$ in Oregon $/ 6 \mathrm{Cl}$ males at $18^{\circ} \mathrm{C}$.) and the effect was quite trivial; the net effect was 96 per cent. of the potential effect. When the final models were considered there were seventeen positive and eight negative nonadditive parameters. This was however not significantly different from equality of sign $\left(\chi^{2}=3 \cdot 24 ; \mathrm{P}=10-5\right.$ per cent.).

Four comparisons between sexes may be made viz. Oregon $/ 6 \mathrm{Cl}$ and Samarkand $/ 6 \mathrm{Cl}$ at $18^{\circ} \mathrm{C}$. and $25^{\circ} \mathrm{C}$. In all four cases the additive effect of the sex chromosome was greater in females than in males although in Samarkand $/ 6 \mathrm{Cl}$ at $25^{\circ} \mathrm{C}$. the difference was not significant. The additive effect of the (hemizygous) sex chromosome in the males was never as low as

TABLE 2

Numbers and effects of non-additive parameters

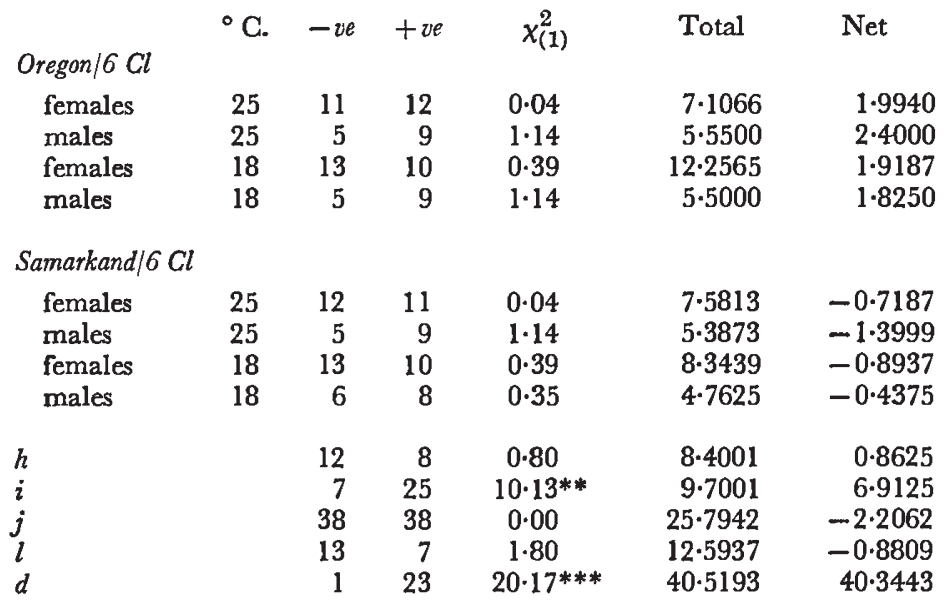

one-half that in females where the sex chromosome was paired, so there would seem to be some compensatory mechanism in males which nevertheless does not fully make up for the missing $\mathrm{X}$ chromosome. The additive effect of the second chromosome did not differ between sexes but that of the third chromosome differed significantly in three cases. This must be ascribed to sex-limitation and has been recorded for additive gene effects controlling body weight in D. melanogaster (Kearsey and Kojima, 1967). The differences between sexes in non-additive parameters can usually be related to a difference in the primary genetic activity of the chromosome. The only exception was found in the Samarkand/6 Cl set at $18^{\circ} \mathrm{C}$. where $i_{23}$ was specified for females and $j_{2 / 3}$ was specified for males. The difference in size of these two parameters was not significant. The difference in action is that in the females the third chromosome acted when homozygous whereas in males the interaction occurred when that chromosome was heterozygous.

In making comparisons between the types of genetical activity found at different temperatures an alternative method of analysis was used. The sum of squares for genotypes of any set of chromosome substitution lines may 
be partitioned into its constituent comparisons (one for each degree of freedom) by conventional orthogonal analysis (Cooke and Mather, 1962; Hill, 1964). This was done, not only for the individual sets, but also for comparable sets of data at the two temperatures. These provide sums of squares for the main effects and for their temperature interactions for every parameter.

The additive effect of the sex chromosome was greater at $18^{\circ} \mathrm{C}$. than at $25^{\circ} \mathrm{C}$. in all four cases, but not significantly so in Samarkand/6 Cl males. It was noted earlier that the total additive effect was greater at $25^{\circ} \mathrm{C}$. than at $18^{\circ} \mathrm{C}$. It follows that the additive effect of the autosomes must have increased with temperature to a sufficient extent to overcompensate for the drop in additive activity of the sex chromosome. Such a situation was found; in the Oregon $/ 6 \mathrm{Cl}$ set the second and third chromosomes had significantly greater additive effects at $25^{\circ} \mathrm{C}$. than at $18^{\circ} \mathrm{C}$. for both sexes. In the Samarkand/6 Cl set the second and third chromosomes in males and the second chromosome in females showed higher additive activity at $25^{\circ} \mathrm{C}$. than at $18^{\circ} \mathrm{C}$. The third chromosome in females was slightly more active at $18^{\circ} \mathrm{C}$. but the difference was not significant (V.R. = 1.002). There seem to be reasonable grounds for saying that the autosomes showed greater additive activity at $25^{\circ} \mathrm{C}$. than at $18^{\circ} \mathrm{C}$. and that the total additive variation followed this pattern even though the sex chromosomes showed less activity at $25^{\circ} \mathrm{C}$. than at $18^{\circ} \mathrm{C}$. In other words the balance of additive genetical activity between the sex chromosome and the autosomes changed in favour of the former at the lower temperature.

Several non-additive parameters also differed significantly with temperature. These were:

Oregon $/ 6 \mathrm{Cl}$ females- $j_{1 / 3}$ and $j_{12 / 3}$

Oregon $/ 6 \mathrm{Cl}$ males- $h_{3}, i_{12}, i_{23}, j_{13 / 2}$

Samarkand/ $6 \mathrm{Cl}$ females $-h_{2}, i_{12}, i_{13}, i_{23}, i_{123}$

Samarkand/6 Cl males- $h_{3}, i_{12}, j_{1 / 2}, j_{12 / 3}$.

All but three of these involve additive genetical effects, which have been shown to change with temperature, so some such changes may be expected. The remaining 59 non-additive parameters did not differ significantly with temperature.

When the data summed over temperatures were analysed the additive parameters accounted for 92 per cent. 95 per cent., 92 per cent. and 95 per cent. of the total sums of squares in Oregon $/ 6 \mathrm{Cl}$ females, Samarkand $/ 6 \mathrm{Cl}$ females, Oregon/6 Cl males and Samarkand/6 Cl males respectively, confirming the very high degree of additive genetical variation for this character.

\section{Discussion}

Whilst some epistatic and dominance parameters were significant it seems reasonably clear that the major genetic component in the control of sternopleural chaeta number is additive. Such non-additive variation as exists is for the most part ambidirectional and therefore self-nullifying though there does seem to be a tendency for additive $\times$ additive interactions to be positive. This is the type of gene action expected for a trait under stabilising selection (Mather, 1966); and Barnes (1968) and Linney et al. (1971) have 
shown this trait to be under that type of selection; flies with intermediate chaeta numbers gave rise to the highest number of progeny and were therefore fitter than those flies with high or low chaeta numbers. It is not clear, however, why the $i$ type interaction should be directional but this was also reported for body weight in Drosophila by Kearsey and Kojima (1967).

The effect of temperature on the gene action seems more marked on the additive than the non-additive parameters (which showed few differences in the full models). This may be contrasted with the work of Law (1966) who grew wheat substitution lines at three temperatures and measured additive effects of chromosomes and the interactions between chromosomes. For the characters studied-height and several components of yield-he found that the interactions varied more than did the additive effects though there was no consistent trend. Law proposed that mutually adjusted effects, such as interactions, would be more liable to disturbance on changing the environment than would the simpler main effects. Since he was able to measure only the total additive effect of all the chromosomes, not their individual effects, a differential but opposing effect of different chromosomes would have gone undetected. Langridge (1962) reviewed several reported instances of heterosis in Drosophila and Arabidopsis and concluded that heterosis became more pronounced at higher temperatures. He suggested that this was due to hybrids withstanding adverse temperatures better than did the inbred parents because populations contained several temperature-sensitive mutants which were exposed in the homozygotes. Lewis (1954) suggested a mechanism based on genotype-environmental interaction to explain heterosis. His model involved the dominance of an allele in a particular environment opposing the effect of that environment so that the $F_{1}$ remained stable. These two authors thus suggest that genotype-environmental interinteraction is a property of particular genes rather than of all the genes contributing to the expression of a trait. In Nicotiana rustica a difference in genotype-environmental interaction for height has been associated with a major marker (Perkins and Jinks, 1968), possibly implying that the genes so affected by the environment are located in that linkage group.

The present study is at the chromosomal, not the genic, level but it does provide experimental evidence that different parts of the genotype affecting the same trait are differentially affected by the environment.

\section{Summary}

1. Two sets of chromosome substitution lines, Oregon/6 $\mathrm{Gl}$ and Samarkand $/ 6 \mathrm{Cl}$ were raised at $18^{\circ} \mathrm{C}$. and $25^{\circ} \mathrm{C}$. and the genetical effects of the three major chromosomes and their interactions were estimated.

2. The additive effects of the chromosomes were found to be much more important than the non-additive effects, the ambidirectional nature of which prevented them making anything but a trivial contribution to the overall differences between genotypes.

3. Increase in temperature increased the total additive effect despite the sex chromosome having a greater effect at $18^{\circ} \mathrm{C}$. than at $25^{\circ} \mathrm{C}$. There were few differences in the non-additive effects at different temperatures.

4. The effect of the hemizygous $\mathrm{X}$ chromosome in males was less than the effect of the paired $\mathrm{X}$ chromosome in females, but not as little as onehalf this effect. There was some sex-limitation of additive effects but 
differences in non-additive effects were usually associated with differences in the corresponding additive effects of those chromosomes.

Acknowledgments.-I am indebted to Dr M. J. Kearsey for his help and advice during the course of this work and to Miss Pat Wilkinson for her technical assistance. The work was carried out during the tenure of a Science Research Council Studentship.

\section{REFERENCES}

BARNES, B. w. 1968. Stabilising selection in Drosophila melanogaster. Heredity, 23, 433-442. COOKE, P., AND MATHER, K. 1962. Estimating the components of continuous variation. II. Genetical. Heredity, 17, 211-236.

HILL, J. 1964. Effects of correlated gene distributions in the analysis of diallel crosses. Heredity, 19, 27-46.

KEARSEY, M. J., AND KOJIMA, x. 1967. The genetic architecture of body weight and egg hatchability in Drosophila melanogaster. Genetics, 56, 23-37.

KELLER, E. C., AND MTTCHELL, D. 1962. Interchromosomal interactions. I. An analysis of morphological characters. Genetics, 47, 1557-1571.

KELLER, E. C., AND MITCHELL, D. 1964. Interchromosomal interactions. II. An analysis of viability characters. Genetics, 49, 293-307.

KILLICK, R. J. 197l. Sex-linkage and sex-limitation in quantitative inheritance. I. Random mating populations. Heredity, 27, 175-188.

IANGRDGE, J. 1962. A genetic and molecular basis for heterosis in Arabidopsis and Drosophila. Amer. Naturalist, 96, 5-27.

LAw, C. N. 1966. Biometric analysis using chromosome substitutions within a species. In Chromosome manipulations and plant genetics (edited by Riley, R. and Lewis, K. R.). Oliver and Boyd, Edinburgh.

IEWIs, D. 1954. Gene-environment interaction; a relationship between dominance, heterosis, phenotypic stability and variability. Heredity, $8,333-356$.

LINNEY, R., BARNES, B. W., AND KEARSEY, M. J. 1971. Variation for metrical characters in Drosophila populations. III. The nature of selection. Heredity, 27, 163-174.

Mather, K. 1966. Variability and selection. Proc. Roy. Soc. London series B, 164, 328-340.

PERKINS, J. M., AND JINKs, J. L. 1968. Environmental and genotype environmental components of variability. IV. Non-linear interactions for multiple inbred lines. Heredity, $23,525-535$.

VAN DER VEEN, J. H. 1959. Tests of non-allelic interaction and linkage for quantitative characters in generations derived from two diploid pure lines. Genetica, 30, 201-232. 